Association for Information Systems AIS Electronic Library (AISeL)

Wirtschaftsinformatik Proceedings 2007

Wirtschaftsinformatik

February 2007

\title{
Supporting Inter-Business Collaboration via Contract Negotiation and Enactment
}

Peter Rittgen

University College of Borås, peter.rittgen@hb.se

Follow this and additional works at: http://aisel.aisnet.org/wi2007

\section{Recommended Citation}

Rittgen, Peter, "Supporting Inter-Business Collaboration via Contract Negotiation and Enactment" (2007). Wirtschaftsinformatik Proceedings 2007. 40.

http://aisel.aisnet.org/wi2007/40

This material is brought to you by the Wirtschaftsinformatik at AIS Electronic Library (AISeL). It has been accepted for inclusion in Wirtschaftsinformatik Proceedings 2007 by an authorized administrator of AIS Electronic Library (AISeL). For more information, please contact elibrary@aisnet.org. 
In: Oberweis, Andreas, u.a. (Hg.) 2007. eOrganisation: Service-, Prozess-, Market-Engineering; 8. Internationale Tagung Wirtschaftsinformatik 2007. Karlsruhe: Universitätsverlag Karlsruhe

ISBN: 978-3-86644-094-4 (Band 1)

ISBN: 978-3-86644-095-1 (Band 2)

ISBN: 978-3-86644-093-7 (set)

(C) Universitätsverlag Karlsruhe 2007 


\title{
Supporting Inter-Business Collaboration via Contract
}

\section{Negotiation and Enactment}

\author{
Peter Rittgen \\ University College of Borås \\ 50190 Borås, Sweden \\ peter.rittgen@hb.se
}

\begin{abstract}
The increasing complexity of products and services encourages more and more companies to form collaborative networks. As these companies are independent organizations there is often an issue of governance. We suggest a possible architecture for such a business network that proposes a contract as the principal means of coordination and describes how such a contract can be designed and enacted.
\end{abstract}

\section{Introduction}

Today we can witness two seemingly opposed trends in the cooperation between businesses: On the one hand companies are forced to concentrate on their core competencies and to outsource all activities that lie outside the core. On the other hand customers demand that a supplier covers an increasing range of products and services. They want to buy a complete solution from only one supplier instead of buying bits and pieces from many. This latter point seems to suggest an increased amount of "insourcing". The solution to both is that companies have to engage in closer cooperations, each concentrating on its area of expertise, but jointly offering a complete suite of related products and services that are well matched (one face to the customer). But this scenario represents an enormous challenge both in terms of organization and regarding the information system support.

Companies that want to engage in a closer cooperation, e.g. a value network, a virtual enterprise or the like, bring into this cooperation not only their different organizational cultures but also 
different, often incompatible, information systems. A successful cooperation therefore requires the alignment or integration of both the business processes and the information systems to a certain degree. In some industries, such as the automotive industry, this can go as far as the customer forcing the suppliers to introduce the ERP system of the customer's choice (e.g. SAP). But on the whole it is more common that the organizations involved will strive for some kind of mutual adaptation of their business processes and information systems. In a very simple case this could be the introduction of a file transfer accompanied by suitable import and export functionalities and some organizational measures for providing and handling the new data. In more advanced cases it will imply substantial reorganization of business processes and changes to existing information systems and/or introduction of new ones.

Our goal is to support the set-up and operation of a business network. The first phase consists mainly of the design of a contract that can be used to coordinate the behavior of network actors. The design process is cooperative, i.e. the actors negotiate this contract among themselves. Such negotiations can be either bilateral or multilateral but both types will contribute to creating one common contract that is binding for all parties involved. We also call this negotiation process a co-design process. Negotiation is a social process that can be supported by a negotiation support system. This eliminates the need for partners to meet face to face and contributes to a flexible set-up of the business network. It implies that the lead-times for setting up the network are relatively short and replacing members that have left and adding new ones can be done with a minimum of effort. These are crucial issues for a business network.

The second phase, operation, consists of enacting the behavior specified in the contract. Here the business logic concerning the coordination of actors is incorporated into the communication network. In this this we phase "translates" from the business network to the communication network by managing the respective message exchange via the technical network and a coordination server. We have used this approach to improve the governance of an existent network that consisted of three partners: the headquarters of a retail chain in the home textile and home decoration industry, the shops of this chain and a third-party logistics provider. Although this is a minimal case of a business network it nevertheless provides fundamental insights into the workings of such networks.

The contribution of our work consists in outlining a method for governing business networks by negotiating and enacting contracts that take the form of business process models. It is based on existing methods for contract negotiation, business process modeling and workflow manage- 
ment. Apart from combining these methods we also enrich them, e.g. by extending the concept of a trade contract to cover process aspects.

The remaining chapters are structured as follows. We first address the issue of coordination in organizational networks in general and business networks in particular, which leads us to the identification of a suitable class of contracts, namely behavior-based contracts. The next sections study the negotiation process and a language for formulating behavioral contracts. We then proceed by describing the enactment of the formalized contract based on a communication network and a coordination server. We conclude this paper by summarizing the major findings and presenting an outlook on future research.

\section{Coordination in a Business Network}

In a business network organizations strive for the provision of complex products and services by coodinating their activities in an "intelligent" way. This implies that the coordination effort is much higher than in a conventional supply chain. In the latter an individual company can focus on managing the relation to a few immediate major suppliers for creating a product or service. In a business network this is not enough but coordination is also required among the suppliers. Theoretically we move from a tree structure to a graph topology which implies that we have to hit a new balance between market and hierarchical coordination. The general problem behind this is quite old and several theories have been advanced to explain the use of a particular form of coordination, most notably Agency Theory [AlchianDemsetz'72; JensenMeckling'76; Ross'73; Wilson'68] and Transaction Cost Economics [Coase'37; KleinCrawfordAlchian'78; Williamson'75, '81, '85]. Based on these theories the internal and external coordination costs can be determined [GurbaxaniWhang'91]. High external costs favour centralization, high internal costs promote decentralization. It is typically assumed that organizations in a supply chain choose their organizational structure and network of trading partners in such a way that the sum of both costs is minimized. There has also been some debate on the impact of information technology (IT). Early work by Malone et al. [MaloneYatesBenjamin'87] suggested that IT will lower transaction costs and therefore, ceteris paribus, lead to an increase in market coordination. Later work posited that organizations will "move to the middle", i.e. to "more outsourcing, but from a reduced set of stable partnerships" [ClemonsReddiRow'93] if non-contractible issues (e.g. quality and trust) play an important role. Empirical evidence [HollandLockett'97] shows 
that companies often operate in a "mixed mode" blending aspects from both markets and hierarchies.

But the majority of these studies was performed in the context of conventional supply chains. In the face of a network topology the balance between hierarchical and market coordination needs to be readjusted: In the absence of a central coordination unit we typically use the contract as an instrument for coordination. Agency Theory suggests two principal forms of contracts, behavior-based contracts and outcome-based contracts. Between an employer and an employee, for example, a contract with a fixed annual salary would be behavior-based as such a contract demands that the agent performs to the best of his capabilities. An outcome-based contract would specify a remuneration that depends on the results that the agent has achieved (e.g. a commission). If the costs for monitoring agent behavior are high, an outcome-based contract is often superior. This is because an unobserved agent is assumed to shirk (i.e. underperform) knowing that he has no consequences to fear. This problem is called moral hazard. An outcomebased contract can be seen as a special case of a behavior-based contract where delivering the outcome is considered to be the only observable behavior of the agent. In addition to that, the costs for monitoring agent behavior have become marginal in many cases due to the omnipresence of information technology. These arguments apply also to the context of business networks. We will therefore focus our investigation on behavior-based contracts.

\section{Negotiation as a Social Process}

We define negotiation as the process whereby a group of two or more individuals tries to reach an agreement on the performance of future actions. The individuals are human beings that might act on behalf of organizations or on their own behalf. For the purpose of this paper we focus on electronic negotiations, i.e. negotiations that are supported by information and communication systems. They can be divided into three different types: bargaining, auction and agent negotiation [KöhneSchoopStaskiewicz'05]. Auctions are very common, especially in electronic commerce. They assume that the traded products or services can be described in detail and are hence comparable. The auction proceeds in the form of a bidding process where potential buyers can make (money) offers for a certain product or service. There are different models to organize the bidding process [Bichler'00]. A comprehensive classification of negotiations with respect to auctions is provided by the Montreal taxonomy [StröbelWeinhardt'03]. Agent 
negotiation means that an inanimate agent, i.e. a software artefact, carries out the process of negotiation on behalf of a principal, typically a human being. The principal delegates the task of negotiating to the agent by providing it with his or her preferences regarding the product or service to be procured. The agent has a certain autonomy to act within the limits of these preferences. Some models for agent negotiation are given in [DignumCortés'01]. The specification of preferences requires that the product or service in question can be described in detail. Hence both auctions and agent negotiation only work with standardized products / services.

The models we have discussed so far assume that most parameters of the contract are already predetermined and very few can actually be negotiated. Most often the only free parameter is the price. In many cases this restriction is not acceptable, i.e. we need more freedom in negotiating. This can, for example, happen if the product or service to procure is not standardized so that we have to negotiate many of its parameters. In such a case we need the third model, bargaining. In bargaining we assume that in principal all parts of a contract are negotiable, i.e. we start with an empty contract (although existing reference contracts or contract templates can be used as a starting point if desired). A number of bargaining models has been suggested such as the Three-Layer Architecture [ChiuCheungTill'03], SilkRoad [Ströbel'01], DOC.COM [SchoopQuix'01], MeMo Business Negotiation Support Metamodel [de MoorWeigand'04], Protocols for Electronic Negotiation Systems [KerstenStreckerLaw'04], and the Generic Model [MathieuVerrons'02]. To find a suitable negotiation model for business networks we must first identify the criteria that it should fulfill. Based on the studies mentioned above we have derived the following criteria: Communication, documents, deontics and time. The next sections argue for the necessity of these criteria.

\subsection{Communication}

The term communication is ambiguous. Communication takes place both on the business network level and on the communication network level but the meanings of the term in these contexts differ fundamentally. In the case of a communication network, communication is the central issue (hence the name). It consists primarily of an exchange of messages between inanimate agents, i.e. computers, IT systems or the like. On the other hand, communication in a business network consists of interaction between human beings (actors). Inanimate agents do not exhibit many of the qualities of human beings, such as conscience, responsibility, creativity and so on. This affects their ability to act as they cannot engage in social action, which requires 
these capabilities. Negotiating a business contract is an example for a complex social process that involves social actions, e.g. making commitments.

Communication is the primary instrument for social interaction in general and for negotiation in particular. Negotiation consists basically of an exchange of messages between the negotiators. With these messages the negotiators create, modify and extend the contract, e.g. by making requests or commitments that ultimately lead to contractual obligations. It is therefore evident that a negotiation model for business network contracts must incorporate communication on a fundamental level. The importance of language for social action has been recognized early which led to the development of several theories, most notably Speech-Act Theory [Austin'62; Searle'69] and the Theory of Communicative Action [Habermas'84]. Many of the negotiation models that address the issue of communication are based on these theories.

\subsection{Documents}

The result of negotiation is a contract, which is obviously a document. Contract and negotiation are duals of each other in the same way that document and communication are. They are so tightly interwoven that it is impossible to separate the one from the other. The contract is a negotiation cast into a document. A negotiation model must therefore provide some mechanism to derive the contract from the negotiation messages in a transparent and traceable way. But documents play an important role already during negotiation. Preliminary contracts (contract versions) are a record of the negotiations that have been made so far. In this sense documents are an embodiment of past communications. We need the contract versions to mark important achievements in contract development, to understand why the contract has developed in that particular way and to go back to an earlier version if something has gone wrong. As this holds for all types of contracts we can conclude that documents must form an integral part of the foundation of a negotiation model for business networks.

\subsection{Deontics}

Deontic logic is concerned with reasoning about obligations and permissions. It has a direct bearing on negotiation as contracts are about determining obligations in exchange for granting permissions. For example, if Henry signs a contract about the sale of a car he enters into an obligation to pay a certain amount of money but in return he is granted the permission to take the car into his possession and to dispose of it in any way he wishes. Deontics trace the status of commitments during the course of a negotiation. As a rule an obligation arises only if all parties 
agree on it. If Sally commits herself to do the shopping she is not yet under any obligation. Mike might, for example, make a counter-offer to do it for her. Only if Mike accepts Sally's commitment is she actually obliged to keep it. The same holds if Sally requests Mike to do the shopping, which he might simply deny. Only his agreement makes it an obligation. Keeping track of the deontic state is therefore important for any negotiation model, especially in the case of the complex negotiations involved in designing the business network.

\subsection{Time}

Time restrictions are an issue for many business actions. Some actions are not allowed to start before a certain point in time, others must be finished before a deadline has expired. A particular action might be required to be performed precisely at a specific time or repeatedly in certain intervals. It is therefore necessary that time restrictions for future actions can be negotiated as they are an important characteristic of the actions. But time-related issues are not only relevant at the level of the business process but also concerning the negotiation process itself. The time order of messages is relevant for the negotiation and the establishments of obligations and there are time limits for the completion of the process. Our negotiation model should therefore offer a language that provides a concept of time.

\section{Architecture of a Business Network}

[KöhneSchoopStaskiewicz'05] has performed an evaluation and comparison of 11 negotiation models with respect to 11 criteria among which the above mentioned criteria can also be found. The closest match to the requirements for a negotiation model is represented by DOC.COM [SchoopQuix'01] which fulfills three of the four criteria fully and one, deontics, at least partially. We have therefore chosen to adopt this model for the purpose of our study. As deontics is an important issue we have decided to add respective functionality to the negotiation system. But there is yet another problem that needs to be solved. The objective of DOC.COM is to represent a negotiation about the execution of a process instance, e.g. the delivery of a particular item on a particular date. But negotiations regarding the set-up and maintenance of a business network concern process types, e.g. the general business logic of order processing. The resulting contract is called a frame contract as it regulates the interaction among network members regarding a significant number of orders over time. To enable such negotiations we 
have introduced a meta-layer into the negotiation language. Figure 1 shows the architecture of a system to set up and operate a business network.

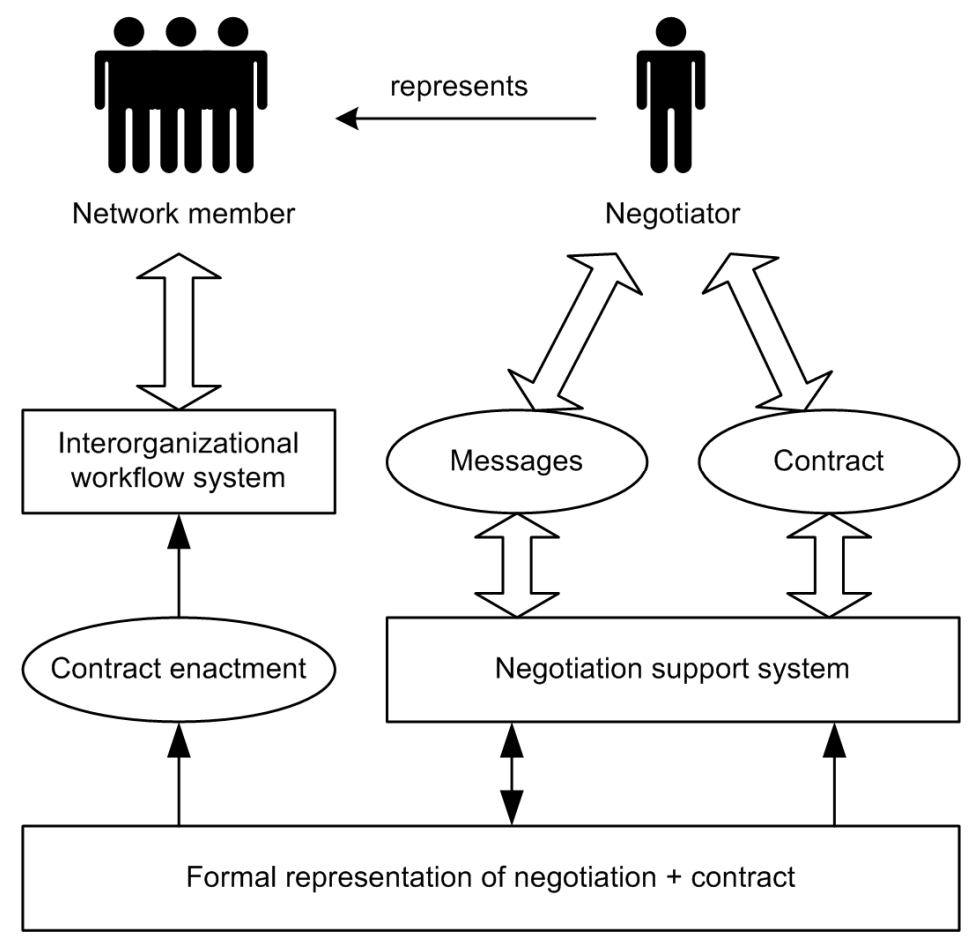

Figure 1: Architecture of a business network

A business network consists of a number of members. Each such member is typically an organization (i.e. a business) but could also be an individual who acts as an economic agent. Each member organization is represented by a negotiator who is entitled to carry out such negotiations and to sign a binding contract on behalf of the organization. This negotiator will interact with negotiators from the other members via a negotiation support system (NSS). The NSS consists of a message component and a contract component. The former handles both the translation of "human" negotiation messages into the formal representation in DOC.COM and the presentation of recorded formal negotiations in a human-readable form. The contract component stores the binding negotiations, which together make up the contract and which are also stored in DOC.COM, and represents this contract in a way that is similar to conventional, written contracts. The specific NSS for DOC.COM is called Negoisst [SchoopJertilaList'03]. The next section describes how negotiation and contract formation proceed.

The left part of Figure 1 shows how the operation of a business network is supported. We assume that the process of negotiation has led to a contract that deals with all relevant issues of the cooperation. This could be the negotiation of a completely new frame contract, i.e. the set- 
up of a new business network. On the other hand the negotiation can also be about business network maintenance which involves adapting to the loss of members, incorporating new members, replacing parting members, reacting to changed requirements or the like. The contract under consideration will in any case be subject to enactment which yields a description of the interactions between the members in some kind of workflow language. The choice of this language depends on the workflow system that we choose to coordinate the workflow between members. In principal any workflow system can be used that allows for the implementation of the workflow patterns identified in [AalstHofstedeKiepuszewskiBarros'03]. Most commercial systems qualify if we allow for workarounds and coding but there is little native support for many of the advanced patterns. Only FLOWer supports directly or indirectly 16 of the 20 patterns. In a prototypical environment it can be useful to employ YAWL [AalstHofstede'05] that provides all patterns but one, together with the YAWL Engine. YAWL uses the same serialization language as the negotiation and contract language DOC.COM, i.e. XML. This facilitates enactment of the contract. YAWL makes also use of XQuery and XPath to extract data from XML input files and for generating XML output. This supports the integration with the enterprise application systems of the business network members, most of which can import and export in XML format. The resulting workflow system is run on a coordination server which can be seen as part of a communication network. An example of this is given in the section 4.3 based on a YAWL implementation. An overview and comparison of other languages for interorganizational workflows is given in [BernauerKappelKramlerRetschitzegger'03].

\subsection{From Negotiation to Enactment}

The previous section has described the general architecture of setting up and operating a business network. In this section we describe how the procedures in that architecture are performed and what the results look like. For this purpose we consider a simple negotiation, the corresponding part of the contract and the resulting workflow net (enactment) in some detail. This example represents only a very small part of the case and just serves to illustrate the way our approach works. The complete example is shown in the next section on a more general level. 


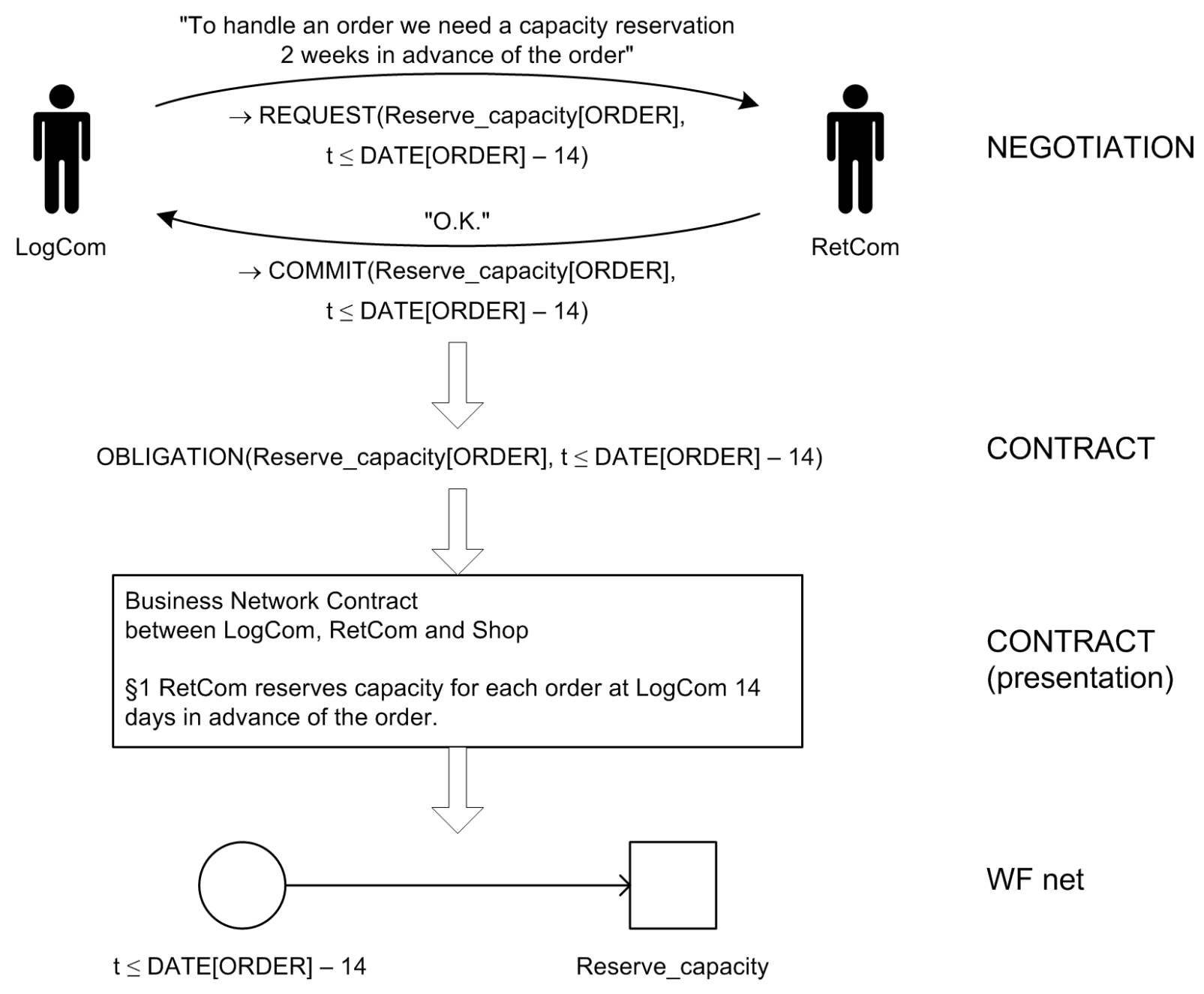

Figure 2: From negotiation to workflow net (example)

Our case involves three business partners: A retail chain in the home decoration industry (RetCom), the shops of this chain and a logistics company (LogCom). RetCom want that LogCom take over the delivery of orders for them. Figure 2 shows two steps in the respective negotiation between them. The representative from LogCom writes an email saying that they need a capacity reservation 2 weeks in advance of the order to be able to handle it. The negotiation support system helps with translating this request from the natural language to the internal, formal representation in DOC.COM:

\section{REQUEST (Reserve_capacity[ORDER], $\mathrm{t} \leq$ DATE[ORDER] - 14)}

The keyword REQUEST indicates that LogCom would like to introduce a new action into their cooperation. The propositional content of this message tells us what that action is, namely the reservation of capacity for each order. The request also specifies a time restriction for this action, i.e. 14 days in advance of the order date. This message is stored in the message memory of the negotiation system so that it can be matched with RetCom's reaction to it. In this case 
RetCom fully agree with the action that was suggested by LogCom by answering with "O.K.". Again the NSS will help with translating this to the formal representation:

COMMIT (Reserve_capacity[ORDER], $\mathrm{t} \leq \mathrm{DATE}[\mathrm{ORDER}]-14)$

The speech act COMMIT signals that RetCom agree to fulfil the request. A request that is followed by a commit with the same propositional content and restrictions leads to a binding obligation of the committing party towards the requesting party with respect to the content. An alternative reaction of RetCom could be:

COMMIT (Reserve_capacity[ORDER], $\mathrm{t} \leq$ DATE[ORDER] - 7)

which would be interpreted as: "We agree to reserve capacity but we cannot do it earlier than one week in advance." Such a speech act does not create an obligation but constitutes a counteroffer. An acceptance of this counter-offer by LogCom would then create an obligation concerning the modified terms. In our example the original request is granted and a respective obligation is inserted into the contract:

OBLIGATION (Reserve_capacity[ORDER], $\mathrm{t} \leq$ DATE[ORDER] - 14)

The presentation component of the NSS can at any point in time display the contract that has been negotiated so far in a human-readable form (see Figure 2). In the final step the obligation is translated to a corresponding workflow.

In the case we have used an orchestration language (YAWL) for that purpose which allowed us to actually implement the coordination processes on a coordination server. The ultimate aim was for LogCom to operate this server and to offer this as an additional service. Another option, from a theoretical perspective, would be to use a choreography language (e.g. WS-CDL) and to decentralize the coordination so that each partner would be responsible for their respective part. In larger networks without any "central" node this is sometimes preferable, especially if the network is unstable. But often companies want to outsource this kind of information logistics in the same way they would outsource physical logistics.

\subsection{Example}

The example in the previous section was on a detailed level but covered only a small part of the case. Here we give a complete account of the case without the details concerning negotiation. We primarily focus on the "old" architecture of the retail network and the result of applying the procedure described in the previous sections with the aim of supporting network governance. We started our project with performing an analysis of the business processes between the companies we have already mentioned. These companies had already an established business 
relationship that was based on a conventional frame contract. In the analysis we discovered the structure of the cooperation (see Figure 3) and a number of problems such as: broken interaction patterns, missing business rules, unclear communication structures, different contract interpretations and excessive interpersonal communication. As a consequence the parties were unsatisfied with the current situation.

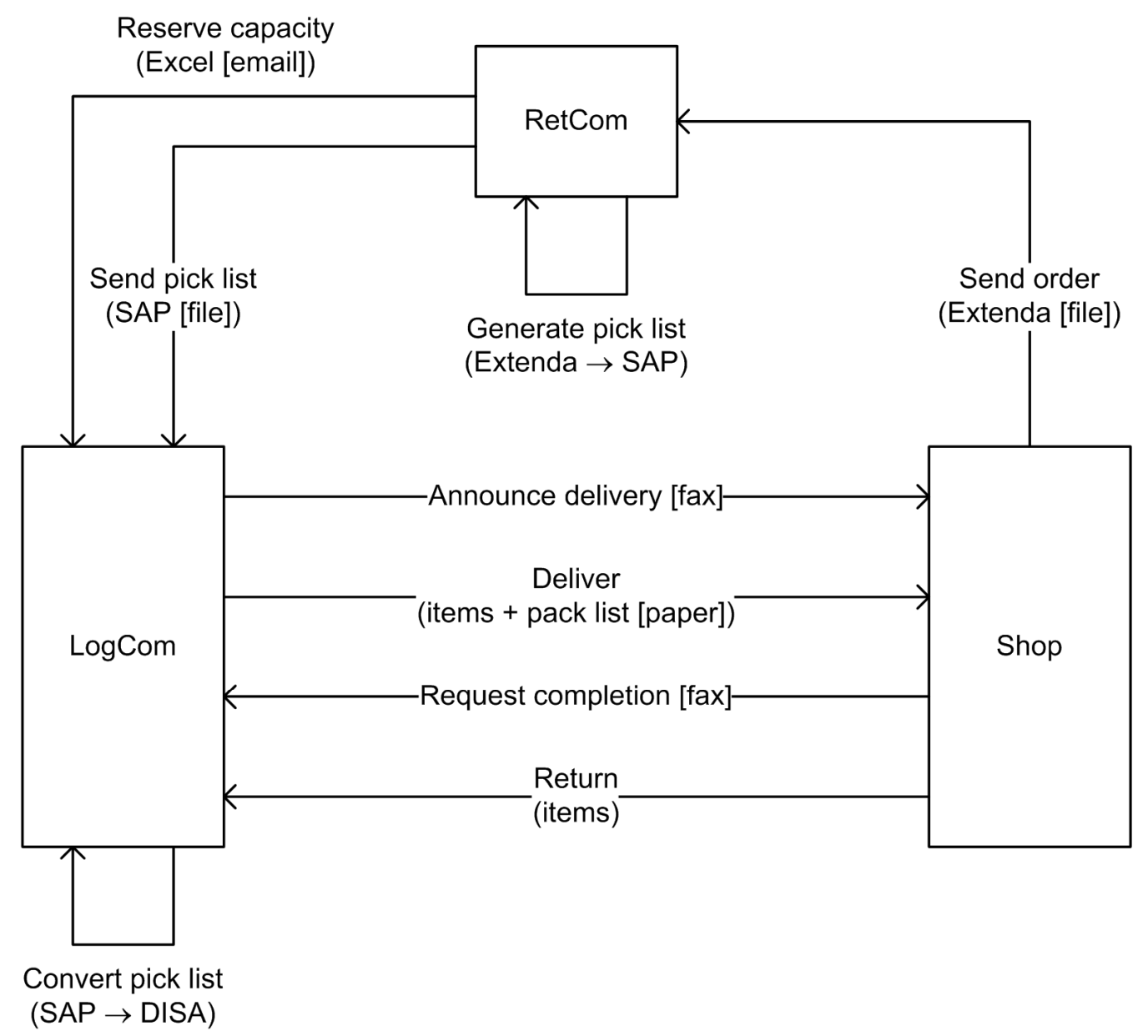

Figure 3: Original architecture of the network

To solve these problems we decided to support the coordination between the network members with the architecture introduced in section 4 . We started with negotiating the formal contract. This was done in a seminar where the representatives of each organization were present and the seminar leader manually translated their requests and commitments into a formal representation according to the procedure described above. The reason for this is that the NSS does so far only support bilateral negotiations. We consider this as a technical restriction rather than a conceptual one and it should be possible to extend the NSS to multi-part negotiation. Enactment of the contract was done with the help of YAWL and the YAWL engine which was run on 
coordination server that conncects all parties. The conversions between the involved formats (SAP, DISA, Extenda and Excel) have been performed with the help of XML Script and the XTract XML Script processor. This led to the architecture depicted in Figure 4.

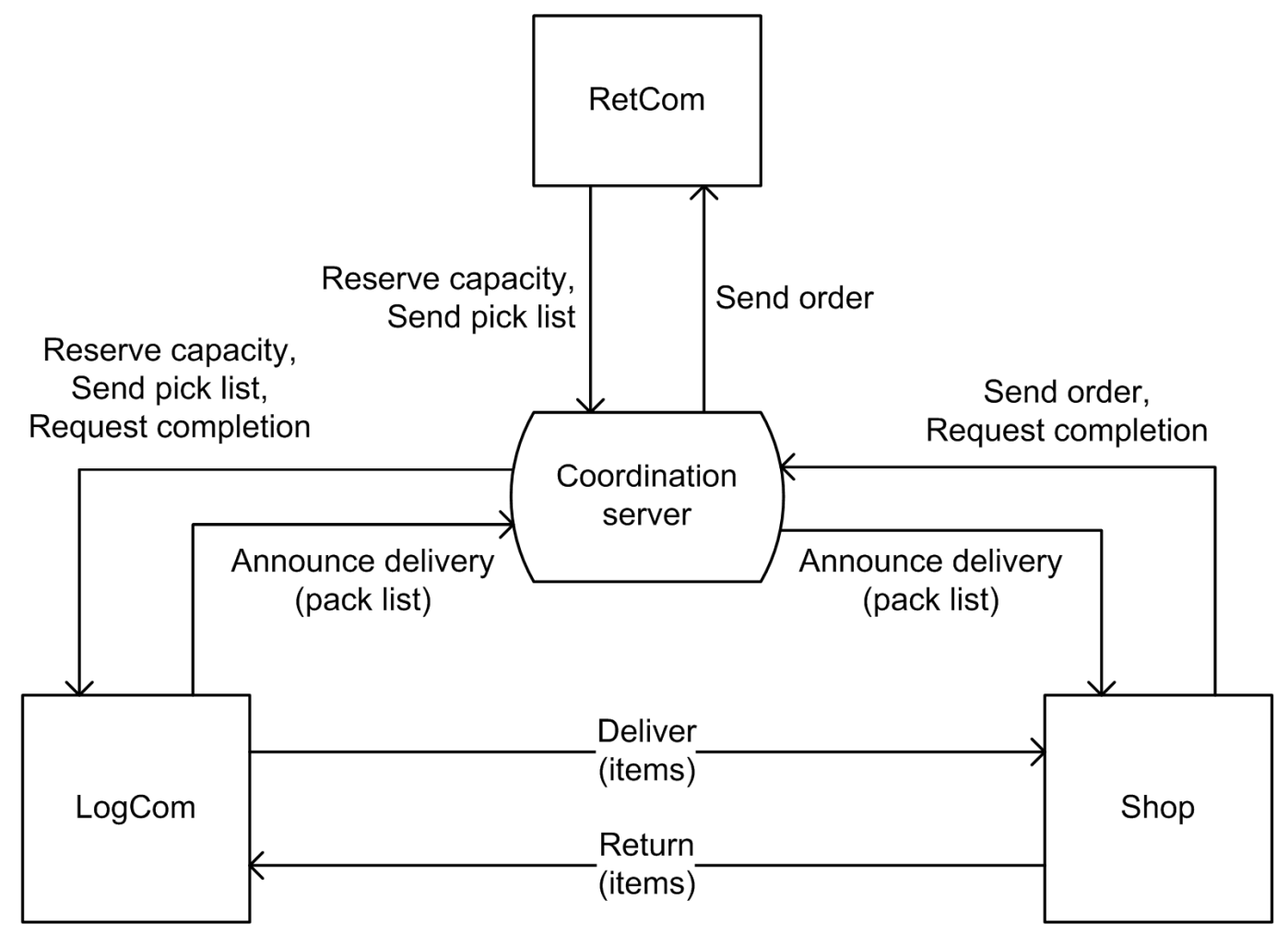

Figure 4: Revised architecture of the network

In the revised architecture each business network partner only exchanges messages with the coordination server. This reduces the complexity of the coordination considerably. The server takes care of forwarding messages to the right recipients, converting between formats, triggering time-controlled messages and so on. The business network architecture also offers ways to improve the efficiency of the communication. In our case, for example, the paper-based communication can be replaced by electronic messages, e.g. concerning the fax containing the pick list. The physical exchange between LogCom and the Shop can in this way be restricted to the exchange of the items themselves. 


\section{Conclusion}

We started from the assumption that a business network consists of a group of businesses that collaborate on an equal footing and coordinate their interaction via a multi-part agreement. Based on relevant theories we indentified a suitable type of contract, i.e. behavior-based, and suggested an architecture to negotiate and enact such a contract. Negotiation is a social process based on interaction between human actors, each of them possibly representing an organization. The nature of social systems and their processes requires that we select an approach that supports human communication as well as documents (as records of human or artefact activity), deontics (as states in the social world) and time. Based on these requirements we arrived at a language for expressing both the process of negotiation and its result, i.e. the contract. This language is DOC.COM. For the enactment of the contract we need an interorganizational workflow management system and a corresponding language. For this step we can so far not make a suitable suggestion as the commercial systems do not provide sufficient support for all required workflow patterns, and the research prototypes do not (yet) possess the maturity and stability required in real-life business applications. To show the feasibility of our approach, we have used it to set up and operate a prototypical business network.

\section{References}

[AalstHofstede'05]

Aalst, W.M.P. van der; Hofstede, A.H.M. ter: YAWL: Yet Another Workflow Language. In: Information Systems 30 (2005) 4, pp. 245-275.

[AalstHofstedeKiepuszewskiBarros'03]

Aalst, W.M.P. van der; Hofstede, A.H.M. ter; Kiepuszewski, B.; Barros, A.P.: Workflow

Patterns. In: Distributed and Parallel Databases 14 (2003) 1, pp. 5-51.

[AlchianDemsetz'72]

Alchian, A.A.; Demsetz, $H$. : Production, information costs and economic organization. In: American Economic Review 62 (1972) 5, pp. 777-795.

[Austin'62]

Austin, J. L.: How to Do Things with Words. Oxford University Press, Oxford 1962.

[BernauerKappelKramlerRetschitzegger'03]

Bernauer, M.; Kappel, G.; Kramler, G.; Retschitzegger, W.: Specification of Interorganizational Workflows - A Comparison of Approaches. Paper presented at the 7th World Multiconference on Systemics, Cybernetics and Informatics (SCI 2003), Orlando, FL, July 27-30, 2003.

[Bichler'00] 
Bichler, M. : A Roadmap to Auction-based Negotiation Protocols for Electronic Commerce. Paper presented at the 33rd Hawaii International Conference on Systems Sciences, Hawaii, 2000.

[ChiuCheungTill'03]

Chiu, D. K. W.; Cheung, S. C. ; Till, S.: A Three-Layer Architecture for E-Contract

Enforcement in an E-Service Environment. Paper presented at the 36th Hawaii International Conference on System Sciences, Hawaii, 2003.

[ClemonsReddiRow'93]

Clemons, E.K.; Reddi, S.P.; Row, M.C. : The Impact of Information Technology on the Organization of Economic Activity: The "Move to the Middle" Hypothesis. In: [Coase'37] Journal of Management Information Systems 10 (1993) 2, pp. 9-35.

Coase, R.H. : The nature of the firm. In: Economica 4 (1937), pp. 386-405.

[de MoorWeigand'04]

de Moor, A.; Weigand, H.: Business Negotiation Support: Theory and Practice. In: International Negotiation 9 (2004) 1, pp. 31-57.

[DignumCortés'01]

Dignum, F.; Cortés, U. (Eds.). (2001). Agent-Mediated Electronic Commerce III. Berlin: Springer.

[GurbaxaniWhang'91]

Gurbaxani, $V$.; Whang, $S$. : The Impact of Information Systems on Organizations and Markets. In: Communications of the ACM 34 (1991) 1, pp. 59-73.

[Habermas'84]

Habermas, $J .:$ The Theory of Communicative Action 1 - Reason and the Rationalization of Society. Beacon Press, Boston 1984.

[HollandLockett'97]

Holland, C.P.; Lockett, A.G. : Mixed Mode Network Structures: The Strategic Use of Electronic Communication by Organizations. In: Organization Science 8 (1997) 5, pp. 475-488.

[JensenMeckling'76]

Jensen, M.C.; Meckling, W.H. : Theory of the firm: Managerial behavior, agency costs and ownership structure. In: Journal of Financial Economics 3 (1976), pp. 305-360.

[KerstenStreckerLaw'04]

Kersten, Gregory E.; Strecker, Stefan E.; Law, Ka Pong Protocols for Electronic Negotiation Systems: Theoretical Foundations and Design Issues. In: Bauknecht, K.; Bichler, M.; Pröll, B. (Eds.): E-Commerce and Web Technologies: Proceedings of the 5th International Conference, EC-Web 2004, Zaragoza, Spain, August 31-September 3, 2004. Springer, Berlin 2004,

[KleinCrawfordAlchian'78]

Klein, B.; Crawford, R.; Alchian, A. : Vertical integration, appropriable rents, and the competitive contracting process. In: Journal of Law and Economics 21 (1978), pp. 297-326.

[KöhneSchoopStaskiewicz'05]

Köhne, F.; Schoop, M.; Staskiewicz, D.: A Meta Model for Electronic Negotiations Comparison of existing approaches. In: Tan, Y. H. (Ed.), Proceedings of the 12th Research Symposium on Emerging Electronic Markets (RSEEM 2005) "Governance of Electronic Markets”. Vrije Universiteit Amsterdam, Amsterdam 2005,

[MaloneYatesBenjamin'87] 
Malone, T.W.; Yates, J.; Benjamin, R.I. : Electronic Markets and Electronic Hierarchies. In: Communications of the ACM 30 (1987) 6, pp. 484-497.

[MathieuVerrons'02]

Mathieu, P.; Verrons, M.-H.: A generic model for contract negotiation. Paper presented at the AISB'02 Symposium on Intelligent Agents in Virtual Markets, Imperial College of [Ross'73]

Science, Technology and Medicine, University of London, 2002.

Ross, S.: The economic theory of agency: The principal's problem. In: American Economic

Review 63 (1973) 2, pp. 134-139.

[SchoopJertilaList'03]

Schoop, M.; Jertila, A.; List, T.: Negoisst: a negotiation support system for electronic business-to-business negotiations in e-commerce. In: Data \& Knowledge Engineering 47 (2003) 3, pp. 371-401.

[SchoopQuix'01]

Schoop, M.; Quix, C.: DOC.COM: a framework for effective negotiation support in electronic [Searle'69] marketplaces. In: Computer Networks 37 (2001) 2, pp. 153-170.

Searle, J. R.: Speech Acts - An Essay in the Philosophy of Language. Cambridge University Press, London 1969.

[Ströbel'01]

Ströbel, M.: Design of Roles and Protocols for Electronic Negotiations. In: Electronic Commerce Research 1 (2001) 3, pp. 335 - 353.

[StröbelWeinhardt'03]

Ströbel, Michael ; Weinhardt, Christof The Montreal Taxonomy for Electronic Negotiations. In: Group Decision and Negotiation 12 (2003) 2, pp. 143-164.

[Williamson'75]

Williamson, O.E.: Markets and Hierarchies. Free Press, New York 1975.

[Williamson'81]

Williamson, O.E.: The modern corporation: Origins, evolution, attributes. In: Journal of Economic Literature 19 (1981), pp. 1537-1568.

[Williamson'85]

Williamson, O.E.: The Economic Institutions of Capitalism. Free Press, New York 1985.

[Wilson'68]

Wilson, R.: The theory of syndicates. In: Econometrica 36 (1968), pp. 119-132. 